\section{e0563 IMPLANTATION OF LEFT VENTRICULAR EPICARDIAL LEAD GUIDED BY OVERLAY REF FOR CARDIAC RESYNCHRONISATION THERAPY}

doi:10.1136/hrt.2010.208967.563

Tang Kai, Zhao Dongdong, Xu Yawei. 'Shanghai 10th People's Hospital, Tongji University School of Medicine

Objective This study was to report our initial experiences with cardiac resynchronisation therapy (CRT) guided by Overlay Ref for the treatment of heart failure.

Methods Eleven patients with heart failure were enrolled in this study, with $(24 \% \pm 11 \%)$ of mean left ventricular ejection fraction (LVEF) and $(78 \mathrm{~mm} \pm 13 \mathrm{~mm})$ of left ventricular end-diastolic dimension (LVEDD). Using Overlay Ref technique, a reference image (inverted) of coronary sinus (CS) and its branches was faded into the live fluoroscopic image. The guidewire (originally designed for percutaneous transluminal coronary angioplasty) could be placed to the target branch of CS guided by Overlay Ref image. Then the left ventricular epicardial lead was positioned to the desired spot through the guidewire.

Results All patients were successfully implanted with LV leads guided by Overlay Ref. Exposure time to X-ray was (19.7 min \pm 14.2 $\mathrm{min})$. And the total duration of procedure was $99 \mathrm{~min} \pm 42 \mathrm{~min})$. 2 weeks after the implantation procedure, ultracardiography (UCG) test showed that LVEF of this patient was raised to $(28 \% \pm 9 \%)$.

Conclusion Overlay Ref technique could facilitate the procedure of LV lead implantation for CRT.

\section{e0564 EFFICACY AND SAFETY OF IMPLANTABLE CARDIOVERTER DEFIBRILLATORAVOIDING ROUTINE DEFIBRILLATION THRESHOLD TESTING}

doi:10.1136/hrt.2010.208967.564

${ }^{1}$ Liu Oiming, ${ }^{1}$ Zhou Shenghua, ${ }^{1}$ Oi Shushan, ${ }^{2}$ Zeng Gaofeng, ${ }^{3}$ Ma Xiaofeng, ${ }^{4}$ Huang He. ${ }^{1}$ Department of Cardiology, Second Xiangya Hospital, Central South University, Changsha; ${ }^{2}$ Department of Cardiology, Second Affiliated Hospital, Nanhua University, Henyang Hunan; ${ }^{3}$ Department of Cardiology, Nanhua Hospital, Nanhua University, Henyang Hunan; ${ }^{4}$ Department of Cardiology, Xiangtan Central Hospital, Xiangtan Hunan

Objective To evaluate the efficacy and safety of implantable cardioverter defibrillator (ICD) and cardiac resynchronisation therapydefibrillators (CRT-D) avoiding defibrillation threshold (DFT) testing when treating ventricular tachycardia (VT) or ventricular fibrillation (VF).

Methods We analysed a continuous database of the 21 patients who had avoided DFT during ICD implantation from Oct. 1999 to Aug. 2008. Follow-up data were completed and analysed in the 21 patients with ICD implantation.

Results ICDs were implanted successfully in 17 patients with VT or VF, and CRT-D were implanted successfully in 4 myocardiopathy patients with severe heart failure who avoided DFT during ICD or CRT-D implantation. Eight patients accepted DFT 1 week later, VT orVF was not induced in 3 patients (37.5\%). During the mean follow-up of $1-7 \quad(4.2 \pm 1.9)$ yrs, malignant ventricular arrythmia was recorded in 16 patients. Among them, 89 episodes were successfully terminated by defbrillation (100\%), 120 VT events were terminated by the first run of antitachycardia pacing $(51.1 \%)$ and 22 by low engery cardioversion (59.2\%). All patients took antiarrhycardia drugs after ICD or CRT-D implantation. No patient died from malignant ventricular arrythmia during the follow-up.

Conclusion No application of routine DFT may aviod complications associated with DFT during ICD or CRT-D implantation. ICD or CRT-D implantation may effectively treat fatal ventricular tachyarrhythmias and prevent sudden cardiac death.

\section{$\mathrm{e} 0565$ ANALYSIS OF INAPPROPRIATE THERAPY IN THE PATIENTS IMPLANTABLE CARDIOVERTER DEFIBRILLATORS}

doi:10.1136/hrt.2010.208967.565

Wang Hui, Qu baiming, Wu Lixuan. Department of Cardiolog Zhejiang Provincial People's Hospital Hangzhou

Objective Observed the efficacy of the patients with implantable cardioverter defibrillators (ICD). Sought to identify the reasons of inappropriate shocks, to minimise the risk of inappropriate shocks. Methods Followed up nineteen patients with ICD. Stored ICD electrograms from all shocks episodes were analysed to distinguish appropriate and inappropriate shocks according to history of tachycardia. Any ICD therapy not delivered for VT or VF was deemed inappropriate shocks therapy. It can be considered as inappropriate that ICD firing for sinus tachycardia, supraventricular tachycardia, atrial fibrillation, atrial flutter, and nonsustained ventricular tachycaidia.

Result There is no shock episode in ten patients (52.63\%). Appropriate shocks occurred in 6 patients (31.58\%) Inappropriate shocks occurred in three patients $(15.79 \%)$. Inappropriate shock episodes constituted 4 of 11 total shock episodes. We presented three cases of inappropriate shocks due to atrial fibrillation, sinus tachycardia and high frequency noise.

Conclusions Inappropriate shocks occurred commonly in our cases. Varied reason induced the inappropriate shocks. Programing the device parameter and choosing proper discriminators can minimised rate of inappropriate shocks.

\section{Q0566 CORRELATION OF ECG FINDINGS WITH SYMPTOMS OF PALPITATIONS USING NOVEL MULTI-LEAD MOBILE PHONE ECG}

doi:10.1136/hrt.2010.208967.566

Michael CL Lim. Singapore Medical Specialists Centre

Objective The aim of this study is to correlate the ECG findings with symptoms of palpitations using a novel multi-lead mobile phone ECG Methods A total of 39 consecutive patients with symptoms of palpitations were enrolled for a period of 3-6 months. Standard 12 lead baseline ECGs were performed and patients were divided into 4 main categories of ventricular conduction defects; 1) sinus tachycardia, 2) supraventricular tachycardia, 3) ventricular ectopics and 4) nonsustained ventricular tachycardia. Patients were each given a mobile phone ECG (EPI Life) to record their ECGs during symptoms. ECG recordings were correlated with symptoms including palpitations.

Results Of the 130 ECG recordings assessed, 33\% showed sinus tachycardia, $25 \%$ supraventricular tachycardia, $41 \%$ ventricular ectopics and 1\% non-sustained ventricular tachycardia. All patients were symptomatic during ECG recordings.

Conclusions Mobile phone ECG (EPI Life) is a useful diagnostic tool for early detection of arrhythmias and prevention of morbidities and mortalities.

\section{Clinical and Research Medicine: Arhythmias} and Intervention Therapy

e0567 EARLY DIAGNOSIS AND RESCUE PERICARDIOCENTESIS FOR
ACUTE CARDIAC TAMPONADE DURING RADIOFREOUENCY
ABLATION FOR ARRHYTHIMIAS, IS FLUOROSCOPY ENOUGH?

doi:10.1136/hrt.2010.208967.567

${ }^{1}$ Huang Xinmiao, ${ }^{1} \mathrm{Hu}$ Jianqiang, ${ }^{2}$ Zhou Fei, ${ }^{1}$ Oin Yongwen, ${ }^{1} \mathrm{Cao}$ Jiang, ${ }^{1}$ Zhou Bingyan. ${ }^{1}$ Changhai Hospital, Second Military Medical University; ${ }^{2}+$ Department of Cardiovascular Diseases, No. 117 Hospital of PLA, Hangzhou, China

Background With the number of complex catheter ablation procedures increasing, procedure-related acute cardiac tamponade is 